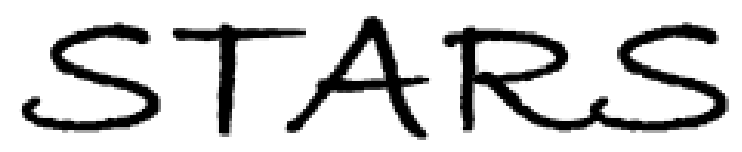

University of Central Florida

STARS

$1-1-1987$

\title{
Magnetic Reconnection Induced By Perturbation On Boundaries
}

Bhimsen K. Shivamoggi

University of Central Florida

Find similar works at: https://stars.library.ucf.edu/facultybib1980

University of Central Florida Libraries http://library.ucf.edu

This Note is brought to you for free and open access by the Faculty Bibliography at STARS. It has been accepted for inclusion in Faculty Bibliography 1980 s by an authorized administrator of STARS. For more information, please contact STARS@ucf.edu.

\section{Recommended Citation}

Shivamoggi, Bhimsen K., "Magnetic Reconnection Induced By Perturbation On Boundaries" (1987).

Faculty Bibliography 1980s. 917.

https://stars.library.ucf.edu/facultybib1980/917

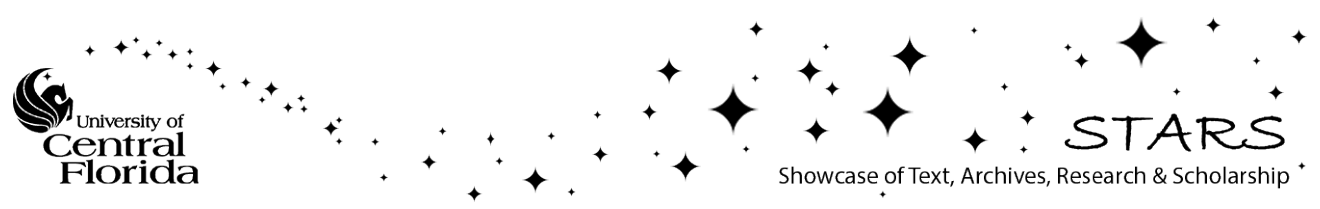


Magnetic reconnection induced by perturbation on boundaries

Bhimsen K. Shivamoggi

Citation: The Physics of Fluids 30, 1228 (1987); doi: 10.1063/1.866276

View online: https://doi.org/10.1063/1.866276

View Table of Contents: https://aip.scitation.org/toc/pfl/30/4

Published by the American Institute of Physics

\section{ARTICLES YOU MAY BE INTERESTED IN}

General theory of the plasmoid instability

Physics of Plasmas 23, 100702 (2016); https://doi.org/10.1063/1.4964481

The interaction of resonant magnetic perturbations with rotating plasmas

Physics of Fluids B: Plasma Physics 3, 644 (1991); https://doi.org/10.1063/1.859863

Tokamak magnetic islands in the presence of nonaxisymmetric perturbations

Physics of Fluids B: Plasma Physics 3, 2617 (1991); https://doi.org/10.1063/1.859974

Nonlinear growth of the tearing mode

The Physics of Fluids 16, 1903 (1973); https://doi.org/10.1063/1.1694232

Tearing mode in the cylindrical tokamak

The Physics of Fluids 16, 1054 (1973); https://doi.org/10.1063/1.1694467

Forced magnetic reconnection

The Physics of Fluids 28, 2412 (1985); https://doi.org/10.1063/1.865247 


$$
\begin{aligned}
& I_{1}=\int_{0}^{1} r J_{0}^{2}\left(p_{0 s} r\right) d r, \quad I_{2}=\int_{0}^{1} r J_{0}^{3}\left(p_{0 s} r\right) d r, \\
& I_{3}=\int_{0}^{1} r J_{0}\left(p_{0 s} r\right) J_{1}^{2}\left(p_{0 s} r\right) d r, \\
& I_{4}=\int_{0}^{1} J_{0}\left(p_{0 s} r\right) J_{1}\left(p_{0 s} r\right) J_{1}^{\prime}\left(p_{0 s} r\right) d r .
\end{aligned}
$$

For discussion we consider the mode $s=1$ for which $p_{0 s}$ $=2.4048$. We propose a possible practical situation with $N_{0} \sim 10^{9} \mathrm{~cm}^{-3}, T_{e} \sim 1.5 \mathrm{eV}, T_{i} \lesssim 0.2 \mathrm{eV}, H \sim 5 \times 10^{3} \mathrm{Oe}$, $a \sim 10 \mathrm{~cm}$. However, we numerically evaluate the coefficients $\delta_{1}, \delta_{2}$ for a wide range of values of plasma density, temperature, and the strength of the external magnetic field that satisfy the assumptions made earlier, i.e., the plasma pressure is small compared to the magnetic pressure and the characteristic time is longer than the ion cyclotron period. As long as $p_{01} c_{s} / a \omega_{i}$ and $p_{01} c_{s} / a \Omega_{i}$ remain $\leqslant 0.1, \delta_{1}$ is found to depend weakly on plasma parameters as well as on the strength of the external magnetic field. Its value is mainly determined by finite geometry and lies between 0.715 and 0.735 . Under such a practical situation, which is supposed to be the usual case, we find it is always permissible to approximate Eq. (13) by the following:

$$
\frac{\partial \alpha}{\partial \tau}+\delta_{1} \alpha \frac{\partial \alpha}{\partial \xi}+\frac{\lambda_{e}^{2}}{2 a^{2}} \frac{\partial^{3} \alpha}{\partial \xi^{3}}=0
$$

where $\delta_{1}$ lies between 0.715 and 0.735 and for the experimental setup proposed above it has the value 0.720 .

In an unbounded medium the nonlinear behavior of ionacoustic waves propagating along an essentially infinite magnetic field may be described by the following wellknown $\mathrm{KdV}$ equation:

$$
\frac{\partial \alpha}{\partial \tau}+\alpha \frac{\partial \alpha}{\partial \xi}+\frac{\lambda_{e}^{2}}{2 L^{2}} \frac{\partial^{3} \alpha}{\partial \xi^{3}}=0
$$

where $L$ is the characteristic scale length of field quantity variations.

Comparing (15) and (16) we find that the main effect introduced by finite geometry enters through the coefficient of the nonlinear term.

The stationary solution to the $\mathrm{KdV}$ equation (13) in a frame moving with a velocity $c=c_{0}+\epsilon A / c_{0}$ is given by

$$
\alpha=\frac{3 A}{c_{0} \delta_{1}} \operatorname{sech}^{2} \sqrt{\frac{A}{4 c_{0} \delta_{2}}}\left(\xi-\frac{A}{c_{0}} \tau\right) .
$$

We define the Mach number as $M=c / c_{0}$. It is now clear from Eq. (17) that, for a fixed amplitude, $M-1$ in bounded plasma in the practical situations mentioned above is smaller by a factor $\delta_{1}(<1)$ than those in unbounded plasma.

\section{ACKNOWLEDGMENT}

The authors gratefully acknowledge the valuable comments of the referee.

${ }^{1}$ H. Washimi and T. Taniuti, Phys. Rev. Lett. 17, 996 (1966).

${ }^{2}$ H. Ikezi, R. J. Taylor, and D. R. Baker, Phys. Rev. Lett. 25, 11 (1970).

${ }^{3}$ E. W. Laedke and K. H. Spatschek, J. Plasma Phys. 28, 469 (1982).

\title{
Magnetic reconnection induced by perturbation on boundaries
}

\author{
Bhimsen K. Shivamoggi \\ Department of Mathematics, University of Central Florida, Orlando, Florida 32816
}

(Received 30 September 1986; accepted 31 December 1986)

The magnetic field reconnection is considered in a plasma induced by perturbing the boundaries of a slab of incompressible plasma with a magnetic neutral surface inside. It is assumed that the boundaries of the plasma slab are perturbed at both fast and slow rates compared with the hydromagnetic evolution rate, and the ensuing adjustments in the plasma and the magnetic field threading through it are investigated.

It is now generally recognized that in geometries that are not perfectly symmetric, the corresponding magnetostatic equilibria will not have smooth magnetic surfaces, but, under the action of boundary perturbations resonating with the field lines on one of the rational surfaces, will lead to the formation and breakup of current sheets into magnetic islands on these rational surfaces. In order to bring out the physical features of this problem, Kulsrud and $\mathrm{Hahm}^{1,2}$ considered a particularly simple model problem that is tractable analytically. Kulsrud and $\mathrm{Hahm}^{1,2}$ studied the magnetic reconnection in a plasma induced by perturbing the boundar- ies of a slab of incompressible plasma with a magnetic null surface inside. The boundaries were perturbed at a rate that was slow compared with the hydromagnetic evolution rate but fast compared with the resistive diffusion rate. Consequent to this perturbation, a new state of equilibrium came into being in which the magnetic field assumed a topology similar to the one before but a current sheet developed at the magnetic null surface. This current sheet brought in resistive effects, which then caused the equilibrium state to evolve into another equilibrium. In the process, a magnetic field reconnection occurred and the current sheet disappeared. 


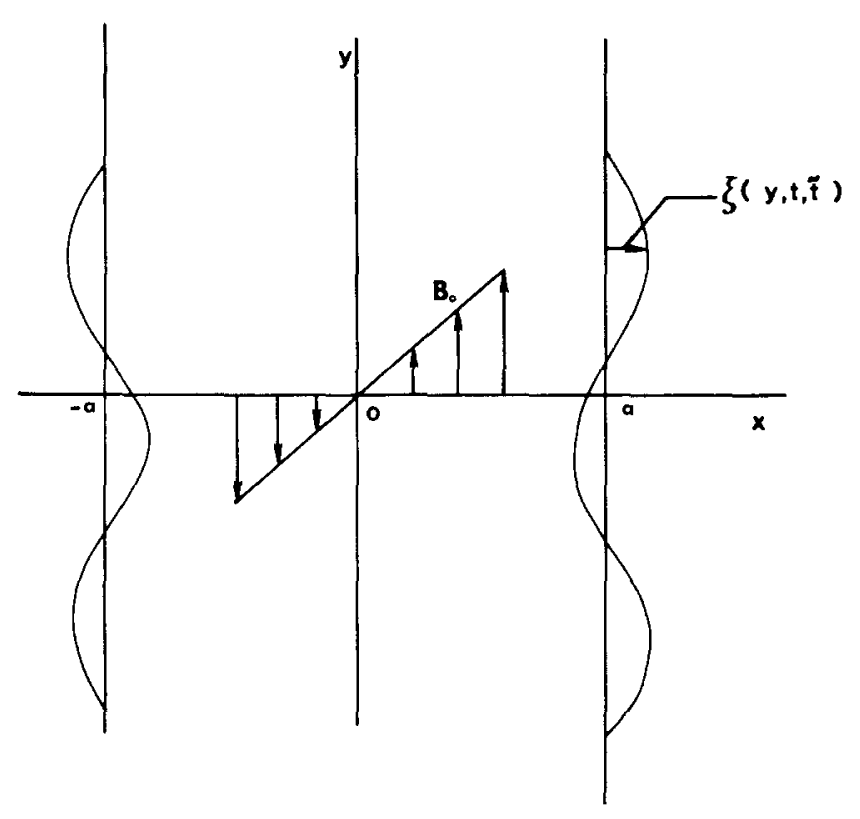

FIG. 1. Plasma slab with a magnetic neutral surface and perturbed boundaries.

This problem has also been considered by $\mathrm{Hu},{ }^{3}$ who instead started with the current sheet as the initial condition. $\mathrm{He}$ found that the ensuing processes then first occur on the Alfvén time scale (which was not surprising because the infinite magnetic field gradient associated with Hu's initial condition would relax at a rapid rate) and subsequently on the tearing mode time scale (as correctly shown by Kulsrud and $\mathrm{Hahm}^{2}$ ).

One question posed by Kulsrud and $\mathrm{Hahm}^{2}$ in this context is whether the magnetic reconnection processes will occur as fast as required by the changing boundary conditions. In this Brief Communication, we propose to answer this question. Intuitively, it would appear that if the boundaries of the plasma slab are perturbed at a rate that is fast compared with even the hydromagnetic evolution rate, one may expect that the current sheet will not develop and that the magnetic reconnection will not occur because these processes occur on the tearing mode time scale and thus would not have time to occur. An interesting problem seems to be when the boundary perturbation has both a variation that is fast compared with the hydromagnetic evolution rate and one that is slow compared with the latter. The ensuing adjustments in the plasma and the magnetic field threading through it are then to be determined. This is dealt with in the present paper.

Consider a plasma in a magnetostatic equilibrium with the magnetic field having a uniform gradient in the $x$ direction (see Fig. 1):

$$
\mathbf{B}_{0}=B_{0}(x / a) \hat{\mathbf{i}}_{y} \text {. }
$$

Let us perturb the boundaries $x= \pm a$ of this equilibrium according to

$$
x= \pm(a+\xi)
$$

where

$$
\xi(y, \tilde{t, \tilde{t}})=\delta(t, \tilde{t}) \cos k y .
$$

Equation (2) shows that the boundary perturbation has a fast variation characterized by the fast time $t$, and a slow variation characterized by the slow time $\tilde{t}=\tilde{\epsilon} t, \tilde{\epsilon}$ being the ratio of the Alfvén time scale $\tau_{\mathrm{A}}$ to the tearing mode time scale $\tau$, and $\tilde{\epsilon} \ll 1$.

Let the velocity of the plasma flow and the magnetic field consequent to this perturbation be given by

$$
\mathbf{v}=\nabla \phi \times \hat{\mathbf{i}}_{z}, \quad \mathbf{B}=\boldsymbol{\nabla} \psi \times \hat{\mathbf{i}}_{z} .
$$

We then obtain, from the equations of plasma motion and magnetic field transport,

$$
\begin{aligned}
& \rho\left(\frac{\partial}{\partial t}+\mathbf{v} \cdot \nabla\right) \nabla^{2} \phi=-\hat{\mathbf{i}}_{z} \cdot\left[\nabla \psi \times \nabla\left(\nabla^{2} \psi\right)\right], \\
& \frac{\partial \psi}{\partial t}+(\mathbf{v} \cdot \nabla) \psi=\eta \nabla^{2} \psi,
\end{aligned}
$$

where $\rho$ is the mass density and $\eta$ is the resistivity of the plasma.

Let $T$ be the time scale characterizing the fast variation of the boundary perturbation $\xi(y, t, \tilde{t})$, and let $\tau$ be that for the slow variation of the latter (which is also taken to be the tearing-mode time scale). Let us write

$$
\begin{aligned}
& \psi(x, y, t, \tilde{t})=\left(B_{0} / 2 a\right) x^{2}+\psi_{1}(x, t, \tilde{t}) \cos k y, \\
& \phi(x, y, \tilde{t}, \tilde{t})=\left(1 / k B_{0} T\right) \phi_{1}(x, t, \tilde{t}) \sin k y .
\end{aligned}
$$

Equations (4) and (5) then give the linearized equations

$$
\begin{gathered}
\frac{\partial \psi_{1}}{\partial t}+\epsilon \frac{\partial \psi_{1}}{\partial \tilde{t}}+\frac{x}{a} \phi_{1}=\frac{T}{\tau_{R}}\left(\psi_{1}^{\prime \prime}-k^{2} \psi_{1}\right), \\
\frac{\tau_{\mathrm{A}}^{2}}{T^{2}}\left(\frac{\partial}{\partial t}\left(\phi_{1}^{\prime \prime}-k^{2} \phi_{1}\right)+\epsilon \frac{\partial}{\partial \tilde{t}}\left(\phi_{1}^{\prime \prime}-k^{2} \phi_{1}\right)\right) \\
=x / a\left(\psi_{1}^{\prime \prime}-k^{2} \psi_{1}\right),
\end{gathered}
$$

where we have nondimensionalized distances using a reference length $L$, and

$$
\tau_{R}=\frac{L^{2}}{\eta}, \quad \tau_{\mathrm{A}}=\frac{\sqrt{\rho}}{k B_{0}}, \quad \epsilon=\frac{\tilde{\epsilon} T}{\tau},
$$

with primes denoting the differentiation with respect to $x$.

Next, the boundary conditions

$x= \pm(a+\xi): \quad \psi=$ const,$\quad \phi_{y}= \pm\left(\frac{\partial \xi}{\partial t}+\tilde{\epsilon} \frac{\partial \xi}{\partial \tilde{t}}\right)$

give

$$
x= \pm a: \quad \psi_{1}=\mp B_{0} \delta, \quad \phi_{1}= \pm B_{0}\left(\delta_{t}+\delta_{t}\right) .
$$

We have

$$
\tau_{\mathrm{A}} / T \gg 1, \quad \tau_{R} / T \gg 1
$$

Let us assume that

$$
\tau_{\mathrm{A}}^{2} \epsilon / T^{2} \ll 1 \text {. }
$$

We then write

$$
\phi_{1}=\phi_{1 f}+\epsilon \phi_{1 s}, \quad \psi_{1}=\psi_{1 f}+\phi_{1 s},
$$

where the subscripts $f$ and $s$ refer to the components that may be identified as those produced, respectively, by the fast variation and the slow variation of the boundary perturbation. Then we obtain, from Eqs. (7), (8), and (10),

$$
\frac{\partial \psi_{1 f}}{\partial t}+\frac{x}{a} \phi_{1 f}=0
$$




$$
\begin{aligned}
& \phi_{1 f}^{\prime \prime}-k^{2} \phi_{1 f}=0, \\
& x=a: \quad \phi_{1 f}=B_{0} \hat{\delta}_{t}, \quad \psi_{1 f}=-B_{0} \hat{\delta},
\end{aligned}
$$

where

$$
\hat{\delta}=\delta-\bar{\delta}, \quad \bar{\delta}=\frac{1}{T} \int_{0}^{T} \delta d t,
$$

from which we have

$\phi_{1 f}=B_{0} \hat{\delta}_{t} \frac{\sinh k x}{\sinh k a}, \quad \psi_{1 f}=-\frac{B_{0} \hat{\delta}}{a} \frac{x \sinh k x}{\sinh k a}$.

Equations (16) show that $B_{1 f y}$ is continuous at $x=0$ so that there is no current sheet at $x=0$. Further, $\psi_{\mathrm{i} f}$ has a topology similar to that of $\psi_{0}$ so that a magnetic reconnection also has not occurred. These results are to be expected because on the fast time scale the current-sheet formation and the magnetic reconnection processes have no time to occur.

Next, we have for the processes on the slow time scale, from Eqs. (7), (8), and (10),

$$
\begin{aligned}
& \psi_{1 s}^{\prime \prime}-k^{2} \psi_{1 s}=0, \\
& \frac{\partial \psi_{1 s}}{\partial \tilde{t}}+\frac{x}{a} \phi_{1 s}=0, \\
& x=a: \quad \phi_{1 s}=B_{0} \bar{\delta}_{i}, \quad \psi_{1 s}=-B_{0} \bar{\delta},
\end{aligned}
$$

from which we have

$$
\begin{aligned}
\psi_{1 s}= & \psi_{1}(0, \tilde{t})\left(\cosh k x-\frac{\sinh k x}{\tanh k a}\right)-B_{0} \bar{\delta} \frac{\sinh k x}{\sinh k a}, \\
\phi_{1 s}= & -\frac{a \psi_{1 \tilde{t}}(0, \tilde{t})}{x}\left(\cosh k x-\frac{\sinh k x}{\tanh k a}\right) \\
& -\frac{B_{0} \bar{\delta} a}{x} \frac{\sinh k x}{\sinh k a}
\end{aligned}
$$

Following the procedure of Kulsrud and $\mathrm{Hahm}^{2}$ we may obtain

$$
\psi_{1}(0, \tilde{t}) \sim \begin{cases}t^{5 / 4}, & \text { for small } t, \\ -B_{0} \bar{\delta} /(\cosh k a), & \text { for large } t .\end{cases}
$$

Using (12), (16), (20), and (21), we have

$$
\begin{aligned}
& \psi_{1}(x, t, \tilde{t}) \\
& \simeq \begin{cases}-\frac{B_{0} \hat{\delta}}{a} \frac{x \sinh k x}{\sinh k a}-B_{0} \bar{\delta} \frac{\sinh k x}{\sinh k a}, & \text { for small } t, \\
-\frac{B_{0} \hat{\delta}}{a} \frac{x \sinh k x}{\sinh k a}-B_{0} \bar{\delta} \frac{\cosh k x}{\cosh k a}, & \text { for large } t .\end{cases}
\end{aligned}
$$

Equation (22) shows that for a generalized boundary perturbation having both fast and slow variations, nothing happens on the fast time scale. However, on the slow time scale, a current sheet develops at small times, magnetic reconnection occurs for large times, and the current sheet disappears. Even on a slow time scale, the fast-varying part of the boundary perturbation has a negligible effect on the reconnection because it goes to zero rapidly as $x \Rightarrow 0\left(\right.$ like $\left.x^{2}\right)$.

'R. M. Kulsrud and T. S. Hahm, Phys. Scr. T2/2, 525 (1982).

${ }^{2}$ T. S. Hahm and R. M. Kulsrud, Phys. Fluids 28, 2412 (1985).

${ }^{3}$ P. N. Hu, Phys. Fluids 26, 2234 (1983). 